\title{
Über die Hörtätigkeit des menschlichen Vorhofes.
}

\author{
Von \\ Dr. O. Goebel, Generaloberarzt a. D. Hirschberg, Schlesien.
}

(Schluß.)

Die Verschiebungsrichtung der Deckhaut folgt, wie bei der M. sacculi, annähernd der Längsachse der M. utriculi. Höchste Töne können diese Macula nicht reizen, weil nur ein geringerer Teil der den Utriculus treffenden Druckdifferenz für die Deckhautveränderung zur Verfügung steht, als beim Sa cculus. Die Einwirkungszeit der Druckdifferenz ist zu kurz. Sehr hohe Töne dürften die Macula zur Tonempfindung erregen. Die Deckhaut muß sich während der ganzen Dauer der +1 leicht umformen können. Das setzt eine nur mäßige Spannung des unteren Utriculusabschnittes am Ende einer +1 Phase voraus. Dessen Anspannung wird nicht allein bedingt durch Wasserversohiebung vom Recessus her, sondern auch durch solche vom oberen Utriculusabschnitt über die häutigen Bogengänge. Fängt der untere Utriculusabschnitt an, sich etwas zu spannen, weiterer Vorbauchung Widerstand entgegenzusetzen, dann findet der Ausgleich der Druckdifferenz in zunehmendem Maße auf dem Perilymphwege oberen-unteren Bogengangsystems statt. Die weitere Umgestaltung des Utriculus wird wesentlich geringer, ein Moment, welches die weitere Umformung der Deckhaut nach dem eben Gesagten verhindert.

Ausreichende Verschiebung der $3 \mathrm{~mm}$ langen Deckhaut verlangt eine etwas stärkere Kraft, - bezogen auf die geringen, zur Verfügung stehenden Druckkräfte, - eine stärkere Kraft, als sie z. B. zur Verschiebung der 1/2-1 $\mathrm{mm}$ breiten Cupüla e erforderlich ist. - Im übrigen gilt für die M. utriculi das bezüglich der M. sacculi Gesagte. (Über die akuztische 
Reizung der M. utriculi bei Zuleitung des Schalles durch den Knochen siehe weiter hinten.)

Der höchste wahrnehmbare Ton würde nur reizen den oberen Teil der Macula sacculi ; bei fortschreitender Tonvertiefung würden der Rei he nach hauptsächlich gereizt werden: unterer Teil der M. sacculi, oberer Teil der M. utriculi, unterer Abschnitt derselben, Schneckenwurzel. Ein bestimmter, sehr hoher Ton würde z. B. erregen den oberen Teil der M. utriculi stark; schwach: den unteren Teil dieser Macula, ebenso den unteren Teil der M. sacculi. Der stetig abnehmenden Schwingungszahl würde eine kontinuierlich sich ändernde Tonempfindung entsprechen, da bei einer bestimmten Tonhöhe z. T. andere Zellen erregt werden als bei einem höheren oder niederen Tone, z. T. die gleichen Zellen in verschiedener Stärke. Die Qualität der Tonempfindung würde auf demselben Prinzip beruhen wie in der Schnecke

Während die + 1 Phase einer tieferen Tonwelle einwirkt, ist Reizung der Maculae durch höchste, bzw. sehr hohe Töne nicht zu erwarten. Die nach der Zisterne zu sehende Oberfläche der Säckchen muß durch die Kräfte der + II Phase erst wieder ihrer normalen Gestalt genähert worden sein, ehe diese Töne gehört werden können. - Bei gleichzeitiger Einwirkung eines tiefen und höchsten Tones besteht die normale Empfindung völliger Glätte des Tones nicht mehr. Dieser ist unruhig, zeigt ein schnelles Vibrieren.

Diese Untersuchungen haben dargelegt, daB Schallwellen an den Macula-Deckhäuten Verschiebungen hervorrufen müs sen, und welcher Art dieselben etwa sind. Danach sind die Maculae durchaus geeignet, Tonempfindungen zu übermitteln. Die Voraussetzung aller hörphysiologischen Betrachtungen war bisher die Annahme: den hin und her gehenden Schwingungen des Schalles mit nach beiden Seiten hingleichem Ausschlag müßten entsprechende Vorgänge der beweglichen Teile des Labyrinthes zugrunde liegen. Diese Vorstellung lag sehr nahe und erschien nahezu selbstverständlich. - Physiologisch wirksam sind die Überdruckphasen; die Unterdruckphasen haben zwar an sich geringere Bedeutung, sind rascheren Annäherung der Organe an ihre Ruhflog ebenfalls unentbehrlich. Sind die Otolithenorgane - * beńto wie die Schnecke - gleichsam Registrierappande Ra bestimmte Drucke, so ist das Verständnis für due doppétite Funktion der Otolithenorgane nunmehr leichter. Als s a tišche Apparate reagieren die Maculae auf Druck und 
Deckhaut, sei es, daß dieser durch Schwere oder durch Trägheit hervorgebracht wird. Die Deckhaut erfährt dabei unter Umständen eine Umgestaltung. Durch Schallwellen geschieht das nämliche. Ergab sich da, bei der Ähnlichkeit der Wirkung, im Laufe der tierischen Entwicklung nichtgleichsam von selbsteine Doppelfunktion? Bei. Gliedertieren, bei Weichtieren mögen die Otolithenorgane nur statische Funktion haben. Je höher das Tier, um so mehr hat sich eine akustische Funktion entwickelt. Bei den Säugern : auf den Maculae nur eine Schicht von Kristallen. Weiter herunter im Tierreich werden es mehrere Lagen; auch werden die Kristalle größer. Bei den Fischen sind es z. T.richtige Steinchen, ebenso bei Gliedertieren, Weichtieren. Diese sind für statische $\mathrm{Z}$ wecke offenbar besser geeignet als die kleinen Kristalle der Säuger.

In welcher Weise kann man sich nun die Differenzierung statischer und akustischer Reize vorstellen? Die auf die Maculae wirkenden statischen Reize sind entweder bedingt durch die Lage des Körpers oder, wie bei den Cristae, hervorgerufen durch Körperbewegungen bez. durch Änderung in der Geschwindigkeit des passiv fortbewegten menschlichen Körpers. Der Reizzustand dauert längere oder kürzere Zeit unverändert an; oder der Reizvorgang spielt sich verhältnismäßig langsam $a b^{1}$ ). Die auf die Maculae wirkenden akustischen Reize, ebenso wie die die Cristae treffenden, sind demgegenüber von wesentlich kürzerer $D$ a uer. Dies Moment kann für Maculae und Cristae zur Differenzierung beider Reizkategorien sehr wohl herangezogen werden. Am einfachsten wäre die Annahme: a kustis che Reize werden durch die Hörborsten auf die Hörzellen übertragen, statische durch die Deckhäute, Cupulae auf die Stützelemente der Nervenendstellen.

1) Shambaugh (Z, f. O. Bd. 65) sieht eins der ursächlichen Momente für die vom Vorhof ausgehende statische Tätigkeit in den Herzpulsationen. Obwohl in den HohIräumen von Vorhof und Bogengängen wohl nur Arterien sehr kleinen Kalibers vorhanden. sein dürften, so müßte doch während eines Pulses der Druck in Vorhof (und Schnecke) ein wenig ansteigen, was minimale Ausbiegung von Basilarmembran und runder Haut herbeiführen könnte. Die an den. Weichgebilden von Vorhor und Bogengängen hierdurch möglicherweise ausgelösten Verschiebungen erreichten ihr Maximum in ungefäh $1 / 4$ bis $1 / 8$ Sek., d. h. sie würden ebenfalls relativ langsam ror sich gehen. Anzunehmen wäre dann, daß die etwa während des Palsmaximums durch Fndolymphverschiebung innerhalb des Utriculas und Sacculus bedingte geringe Anspannung der unteren Abschnitte dieser Bläschen die aluustische Funktion nicht beeinträchtigte, d. h. daß während des Pulsmaximums diese Abschnitte weitere Anspannung mit Leichtigkeit zuließen. 
Hierfür scheint ein histologisches Moment zu sprechen. In der Schnecke umgibt das Nervengeflecht nur den unteren Pol der Hörzellen, in den Vorhofsnervenstellen auch deren Längsseiten $(\text { Held })^{1}$ ). Nervenfasern reichen bis an die Grenzmembran (Held). Ein Kontakt der aufsteigenden Fasern mit den Stützzellen ist nicht ausgeschlossen (v. Ebner in Köllickers Gewebelehre). Es könnten zwei differente Nervenplexus vorhanden sein: der oberflächlichere für die statischen, der tiefere für die akustischen Reize.

Für die Differenzierung beider Funktionen an der Ma culae wäre freie Endigung der Hörborsten in den Deckhauthohlräumen vorteilhafter. Druck oder $Z u g$, senkrecht a uf die Fläch e einer Macula wirkend, ruft im wesentlichen nur Druckunterschiede hervor, keine Verschiebung. Die Hörborsten bleiben unbeeinflußt. Eine auf die Deckhaut ihrer Dicke nachwirkende Kraft (Schwere, Trägheit) verschiebt die ganze Deckhaut gleichmäßig um eine gewisse Größe; die Hörstäbchen werden, bei ihrer Lage in Hohlräumen, entweder gar nicht verschoben oder in Richtung der Deckhautverschiebung ein wenig gebogen. Biegung der Höhrstäbchen ruft an der Hörzellenoberfläche andere Veränderungen hervor wie Druok auf diese, braucht sonach nicht unbedingt erregend zu wirken - abgesehen davon, daß der Vorgang langsam erfolgt.

Werden auch die statischen Reize auf die Hörzellen übertragen, so müßte die Differenzierung dieser und der akustischen Reize in dem sie umspinnenden Nervenplexus oder weiter zentralwärts, z. B. in subkortikalen Ganglien erfolgen. -- Gleiches würde für den Fall gelten, daß die Cristae keine organisierte Cupula besitzen.

Die Maculae sind wahrscheinlich verantwortlich zu machen für die dauernden Augenverstellungen bei bestimmten Veränderungen der Kopfstellung und für die beim Karussellfahren auftretende Sinnestäuschung: mit dem Körper nach außen hin geneigt zu sein. Die statischen Nerven der Maculae werden also in verschiedener Weise erregt, je nach der Wirkung, die Schwere oder Trägheit - unter verschiedenen Bedingungen - auf die Otolithen ausüben. Diese Möglichkeit, daß Trägheit und Sehwere in verschiedener Weise die statischen Maculanerven erregen können, läßt sich meines Erachtens am leichtesten erklären, wenn die Hasse-Retziussche Dar-

1) Held, Untersuchungen über den feineren Bau des Ohrlabyrinthes der Wirbeltiere. 
stellung über die freie Endigungsweise der Hörstäbchen in den Deckhauthohlräumen zu Recht besteht. Befinden sich eine Macula horizontal unter oder über ihren Otolithen, so üben diese gleichmäßig auf deren ganze Fläche einen gewissen Druck oder Zug aus. Alle ihre statischen Nerven werden in gleicher Weise beeinflußt. Wirkt die Schwere ein parallel zur Ma c ula oberfläche, so wird die gesammte Deckhaut in einem nach ihrer freien Fläche zunehmenden Maße in der Schwerkraftrichtung verschoben. Dem Deckhautzuge folgen ein wenig die mit ihr - aller Wahrscheinlichkeit nach - organisch verbundenen Stützzellen, während die Hörzellen hierdurch nicht unmittelbar beeinflußt werden. Oberhalb einer Hörzelle (im Sinne der Kraftrichtung) werden die Stützzellen, bzw. deren der Maculaoberfläche angrenzenden Teile, der Hörzelle um ein Minimum genähert. Die dazwischen befindliche nervöse Interzellularsubstanz wird einem minimalen Dr uc ke ausgesetzt, während unterhalb dieser Hörzelle das umgekehrte geschieht. Die oberhalb und unterhalb der Hörzelle befindlichen Nerven werden - in verschiedener Weise - gereizt, während die zurzeit rechts und links von ihr verlaufenden Nervenfasern vielleicht gar nicht oder schwächer erregt werden, da hier im wesentlichen nur minimale gleichartige Verschiebung der Stützzellen erfolgt, ohne daß zwischen ihnen Druck oder Zug entstände.

Häufig wurde bei absoluter Taubheit ein ,normaler" histologischer Befund an den Vorhofsnervenendstellen angetroffen. Hieraus einen Schluß ziehen zu wollen auf das Fehlen einer akustischen Vorhofsfunktion ist nicht angängig. Über die Endigungsweise der Hörborsten an den Maculae, Cristae wissen wir nichts Sicheres. Ob auf den letzteren eine organsierte Cupula aufsitzt oder.strukturlose Gallert, ist ebenfalls noch nicht in allgemein anerkannter Weise entschieden. Das sind sehr wichtige Dinge für die Funltion dieser Organe. In den allermeisten Fällen sind zudem die Vorhofsnervenstellen beim Menschen durch postmortale Vorgänge so weit verändert, daß Feststellung feiner Details ausgeschlossen ist. Wir sind nicht in der Lage, bei einer menschlichen, in pathologischer Hinsicht zu beurteilenden Nervenendstelle des Vorhofes sagen zu können: sie sei ,normal". Dazu müßten alle feinen Details sicher bekannt, und wir in der Lage sein, an dem zu beurteilenden Präparat deren Vorhandensein oder Fehlen sicher festzustellen. Wenn auch Nervenfasern, Epithelzellen normal erscheinen : an den Hörzellen, den Deck- 
häuten können minimale, nicht nachweisbare Veränderungen bestehen, die die gröbere statische Funktion noch zulassen, die feinere, akustische ausschließen. Ist die Cupula oder Deckhaut mit den Hörhaaren abgehoben oder stärker verschoben: so ist das für die Funktion Wesentliche zerstört ${ }^{1}$ ).

Ich möchte das eben Gesagte durch ein Beispiel erläutern: Taubheit - mit hohen subjektiven Tonempfindungen - könnte bestehen bei einer leichten Schrumpfung der Deckkaut. Die Hörborsten würden dann - nach der Retziusschen Auffassung über ihre Endigung - dauernd gedrückt werden, mithin subjektive Tonempfindung auslösen. Den durch Schallwellen bedingten Kräften würde nennenswerte Verschiebung der Deckhäute nicht mehr gelingen, wohl aber noch in beschränktem Maße den gröberen Einwirkungen von Schwere und Trägheit. Wären, etwa durch Abnormitäten des Lymphstromes, die DeckhauthohIräume erweitert, so könnte unter Umständen kein Druck mehr auf die Hörborsten ausgeübt werden; Taubheit wäre die Folge. Statische Einflüsse könnten die Deckhaut noch verschieben. - Nach Breuers Auffassung über die Endigung der Hörhaare könnte das letzte Moment bedingen: Taubheit mit subjektiven Tönen, durch dauernde Anspannung der Haare das erste Moment: Taubheit; eine Anspannung der Haare wäre nicht mehr möglich.

Ich gehe über zur a kustischen Funktion der Bogengänge. Wir müssen mit der Möglichkeit rechnen, daß eine Cupula auf den Kristen vorhanden ist oder fehlt. Im letzten Fall ist das Bestehen einer gallertigen strukturlosen Zwischensubstanz zwischen den einzelnen Hörhaaren anzunehmen, eine Conditio, sine qua non, um Verwirrungen der langen Haare durch Lymphströnzungen zu verhüten, andererseits ein Moment, welches die Bewegung der Borsten durch schwache Kräfte fördert. - Ist eine organisierte Gallerthaube vorhanden, - eine Annahme, in der ich Bre uer folge -, so liegen zwei Möglichkeiten vor hinsichtlich des Verhältnisses von Deckhaut (Cupula) und Hörborsten : diese enden frei, sind bis an die Spitze von einem Hohlraum umgeben, oder sie gehen über in das Deckhautgewebe. Die die Haare umgebenden Hohlräume (Hasse, Retzi us) müssèn nach den Haarspitzen zu recht eng werden. - Veranlaßt wird das durch die - im Am-

1) Nach Wittmaack (Z. f. O. Bd. 65) ist Abgehobensein der Otolithenmembran oder Cupula für menschliche Präparate geradezu das Normale. Vorhandensein einer fest aufsitzenden Deckhaut (Cupula) läßt auf das Vorliegen degenerativer Prozesse schlieBen. 
pullenquerschnitt - stark kon kave Kristenoberfläche, durch die demzufolge vorhandene Konvergenz der in einer Querschnittsebene (der Ampulle) stehenden Borsten und durch deren verhältnismäßìg sehr bedeutende Länge: - $\mathrm{Ob}$ die Spitzen der Borsten frei endigen, von einem min i ma I en Hohlraum umgeben oder sich mit der Haube organisch verbinden : in beiden Fällen müssen sie alle Verschiebungen der Deckhaut mitmachen. Für den Bewegungsvorgang an sich ist es auch ohne Bedeutung, ob die Haare in strukturlose Gallert oder in ein gallertiges Gewe be eingebettet sind.

Die Bindegewebsfalten der Kristen liegen für die Remanenz außerhalb des Bereichs der stärksten Verschiebung. Auch bieten sie viel mehr Widerstand als die Hauben. An jenen wird die Verschiebung durch Remanenz, wie durch die seitens der Schallwellen im Vorhof ausgelösten Druckdifferenzen reoht gering sein. Die Bewegung beschränkt sich im wesentlichen auf Haare und Hauben. Diese werden beim Begleichen einer jeden, im Vorhof auftretenden Druckdifferenz - sofern dies vermittels der häutigen Bogengänge geschieht, - in Richtung der Unterdruckszone verschoben.

Sham baugh (Z.f. O., Bd. 65) ist der Meinung: Die Cupula werde durch den Stoß der Endolymphströme nur auf der Überdruckseite ,eingedrückt", also nicht als Ganzes verschoben. Trifft einseitiger Überdruck die Cupula, die als ein sehr weiches, daher in mäBigen Grenzen leicht verschiebliches Gebilde anzusehen ist, so wird zunächst der auf der Überdruckseite gelegene Teil ein wenig verschoben, und zwar sowohl vom Rand der häutigen Ampulle zu deren Mitte, wie von der Kristenhöhe zur Cupulaspitze in zunehmendem Maße. Der beweglichste Teil der Cupula ist also der mittlere Teil ihres freien Randes. Durch die Annäherung der zunächst getroffenen Molekularebenen an ihre in der Kraftrichtung folgenden Ebenen wird zwischen beiden molekularer Überdruck erzeugt, der letztere in gleicher Weise wie erstere verschiebt. Bei dem geringen Dickendurchmesser der Cupula (zirlka $1 / 2-1 \mathrm{~mm}$ ) ist ein isoliertes Eindrücken einer Fläche nicht möglich ohne gleichzeitige entsprechende Vorbauchung der Gegenseite.

Beim Vorhandensein einer organisierten Cupula könnte man sich die Differenzierung akustischer und statischer Reize folgendermaßen vorstellen: Wie bei den Maculae, verschiebt Biegung der Cupula die Stützzellen bzw. deren dor Oberfläche angrenzende Teile um ein Minimum in der Kraftrichtung, 
nähert diese also den Hörzellen, die von der Cupulaverschiebung nicht unmittelbar betroffen werden. Während die $\mathrm{Cu}-$ pula ihrer ganzen Höhe nach von einseitigem Überdrucke betroffen wird, werden nur die oberen Abschnitte der Hörborsten von der sich bewegenden Cupula mitgenommen, gleichgültig, ob diese, von engen Hohlräumen umgeben, frei endigen oder sich organisch mit dem Cupulagewebe verbinden. Auf die unteren Hörborstenteile, die in weiteren Hohlräumen stecken, wirkt die Cupulaverschiebung nur mittelbar durch Bewegung der Hohlraumlymphe. Der Querschnitt dieser Hohlräume wird durch die Dehnung der Cupula ihrer Länge (Breite) nach ein wenig in dieser Richtung verbreitert, so daß die Flüssigkeit seitlich der Haare ausweichen kann, daher nur geringeren Druck auf diese ausübt. Die Hörzelle, bzw. ihr der Oberfläche benachbarter Teil, wird jedenfalls weniger oder gar nicht in der Kraftrichtung verschoben, zumal die Kraft nur an einen kleinen Teil der Zelloberfläche angreift - (im Gegensatz zu der Wirkung der Cupula auf die Stützzellen). Die Hörzelle erfährt nur an jhrer Oberfläche eine geringe Veränderung: Dehnung auf seiten der Haarkonvexität, Druck auf seiten der Haarkonkavität. Wie an den Maculae wird die nervöse Interzellularsubstanz zwischen Hör- und Stützzellen auf der $\ddot{U}$ berdruckseite um ein Minimum komprimiert, auf der Unterdruckseite gedehnt. Hierdurch würde eine verschiedenartige Reizung der statischen Nerven auf der Über- und Unterdruckseite jeder Hörzelle gegeben sein.

Die Hörzellen der Kristen werden a ku stis ch gereizt durch rasche Biegung der Hörborsten, gleichgültig, in welcher Richtung das geschieht. Langsame Biegung von Cupula und Hörborsten würde die Hör zellen unerregt lassen, erregte dafür die zwischen Stützzellen und Längsseiten der Hörzellen befindliche nervöse Interzellularsubstanz.

Resonanz im engeren Sinne, d. h. ein allmähliches Verstärken der Schwingungen einzelner Haare unter Einwirkung bestimmter Wellenlängen über die Verschiebung des umgebenden Wassers hinaus, ist auszuschließen. Das spezif. Gewicht der Borsten ist zu gering, als daß ihre Schwingungen die nötige Energie haben sollten, sich durchzusetzen gegen den Widerstand der sich hin und her verschiebenden Flüssigkeit, noch dazu im engsten Raume. Die Haube (Schleimschicht) müßte eine etwa vorhandene Tendenz zum Mitschwingen der Haare lahmlegen durch ihre - spontan aller Wahrscheinlichkeit nach langsam zurückschwingende 
Masse. Die Bewegungen von Haaren und Hauben sind keinesfalls stärker wie bei der Endolymphe, wohl aber schwächer, - da erstere größeren Widerstand leisten als letztere.

Bei den Remanenzvorgängen spielt sich der Hauptteil der Verschicbung in den häutigen Bogengängen ab. Die Perilymphräume sind an den glatten Kanalenden häutig verschlossen; da sind nur minimale Verschiebungen möglich. Auch die akustischen Verschiebungen im äuBeren Bogengang geschehen der Hauptsache nach im häutigen Gange, wogegen im oberen-unteren Kanalsystem hierfür auch die Perilymphräume benutzt werden.

Die häutigen Kanäle sind beim Menschen sehr dünnwandig $(0,009-0,012 \mathrm{~mm}$ Retzius $)$. Entsteht im Perilymphraum oder im häutigen Kanal relativer Überdruck, so kann der sich umsetzen in geringe Verengerung oder Erweiterung des Lumens häutigen Bogenganges.

Im oberen häutigen Bogengange ist die wirksame Druckdifferenz (+ I Phase; Luftleitung) am größten; sie führt zur Verschiebung von Wasserteilchen nach der Öffnung des Crus commune hin und zur Öffnung der unteren häutigen Ampulle. Im unteren häutigen Gange ist daher die Druckdifferenz geringer, - geringer auch als im äuBeren häutigen Gange. In letzterem dürfte sie ein wenig kleiner sein als im oberen Gange.

Folgendes geht im Utricular-Bg.-System vor während der Druckdifferenz, die eine + I (Luftleitung) im Vorhof auslöst:

Im horizontalen Bg. erfolgt die nach dem glatten Kanalende zu gerichtete Verschiebung überwiegend im $\mathrm{häu-}$ tigen Gange. Im Perilymphraum bleibt der Druck etwas höher als im häutigen Kanal, da in jenen der relative Unterdruck erheblich schwerer als in diesen eintreten kann. Das führt zu einer Lumensverkleinerung des häutigen Ganges, die natürlich, den geringen Kräften entsprechend, sehr gering ausfällt. Immerhin wird ein Teil der an beiden Kanalenden herrschenden Druckdifferenz hierdurch beglichen. Die Endolymphverschiebung im Ampullenabschnitt des häutigen Ganges wird geschwächt, damit die Versohiebung der Haube mit den Haaren.

Die Verbindung der Zisterne des Vorhofes mit den Perilymphräumen der drei Ampullen ist genügend weit. Die häutigen Ampullen füllen die knöchernen nur bis zur Hälfte aus 
(Siebenmann $)^{1}$ ). - Im oberen-unteren Kanalsystem gleicht sich die Druckdifferenz zwisehen Dach und Grund des Vorhofes aus durch die häutigen wie durch die Perilymphkanäle. Die Perilymphe des unteren Bg.s verschiebt sich leicht. Die Verschiebungsrichtung geht um das Dach der häutigen unteren Ampulle herum nach dem Vorhofsgrunde. Die Perilymphe übt hier keinen Druck aus auf den häutigen unteren $\mathrm{Bg}$.

Der Überdruck im Perilymphraum oberen Ganges erzeugt Verschiebung nach dem Perilymphraum unteren Ganges. Ehe aber der Unterdruck im Perilymphraum oberen Ganges wirksam wird, hat sich der Druck im häutigen oberen Kanal schon ein wenig erniedrigt. Das bedingt minimale Lumensverengerung häritigen oberen Ganges, die aber geringer ist als am äußeren Gange.

Während der + II und - I Luftleitung herrseht Überdruck an den drei unteren, Unterdruck an den beiden oberen Bogengangsöffnungen. - Die Vorgänge in den Bogengängen sind entgegengesetzt den für die + I Phase geschilderten. -

Voraussetzung für die Endolymphverschiebung, für die Bewegung der Cupula ist: Nachgiebigkeit des unteren Utriculusabschnittes. Ist der bereits über ein besimmtes Maß hinaus gespannt, dann hört die Endolymphverschiebung im Utriculus-Bogengangssystem auf, findet nur noch im oberen-unteren Perilymphkanalsystem statt.

Eine derartige Anspannung des unteren Utricularabschnittes würde bald nach dem Einsetzen der $+\mathrm{I}$ einer kräftigen, langen Welle eintreten, wenn die häutigen Bogengänge direkt vom Knochen umschlossen wären. Die Verbindung der Perilymphräume oberen-unteren Bogens schafft einen Nebenweg, der das Eintreten zu starker Utriculusspannung verzögert. - Wenn lange + I Phasen einwirken, so kann gleichwohl in ihrem Verlauf die Utriculusspannung zu groß werden. Erst nach. Rückgang der Spannung in der + II wäre dann wieder Lymphverschiebung im Utricularsystem möglich. Lange + I Phasen könnten also in ihrem 1. Teil Hörwirkung erzielen, aber nieht mehr in ihrem Endabschnitt. Wellenlänge als Erregungs- und Differenzierungsprinzip wäre hier von geringer Bedeutung.

1) Hb. d. Anatomie von v. Bardeleben. 
Wie kann man sich die physikalischen Vorgänge bei Entstehung von Reibegeräuschen vorstellen? Streiche ich mit dem Finger über eine Bürste, so höre ich kürzeste Tonstöße verschiedener Höhe neben $h$ - und f-ähnlichen Geräuschen. Die verschieden langen, verschieden elastischen Borsten folgen dem. Zuge des Fingers bald mehr, bald weniger, bald gar nicht. Unausgesetzt kommt es hierbei, wie beim Zurückschwingen der Haare, zu Zusammenstößen einzelner Borsten. Ein Tohuwabohu der verschiedensten, nebeneinander laufenden, einander folgenden Wellen und von Wellenfragmenten entsteht, welch letztere durch Interferenz geschaffen und in der verschiedensten, wechselndsten Art zusammengesetzt sind. Proteusartiger Wechsel der Schwingungsformen und -phasen beherrscht in jedem Moment die Szene. Wenn auf die Schnecke nur regelmäBige Schwingungen einer Tonhöhe einwirken, dann ist die Tonempfindung am reinsten. Schon wenn zwei differente Töne gleichzeitig die Schnecke treffen, ist die Reinheit der Empfindung erheblich gestört. Sie wird unruhig. Sekundäre Tonempfindungen treten auf.

Wenn auch die Schnecke durch die komplizierten Schwingungsformen von Reibegeräuschen zu momentanen und sehr differenten, wechselnden, rudimentären Tonempfindungen erregt werden kann, so ist man gleichwohl berechtigt, die Schnecke als weniger geeignet hinzustellen für die Auffassung von Reibegeräuschen wie von Tönen. Das Erregungs-, Differenzierungsprinzip bei der Schnecke ist die Wellenlänge, mit der die Ablaufart der einzelnen Welle gegeben ist. Da, wo in der physikalischen Erscheinungsform des Schalles die Wellenlänge eine nur untergeordnete Rolle spielt, ist die Schnecke gewissermaßen außerstande, den Charakter solcher Schwingungsgebilde in der Emptindung durch Tonfragmente wiederzugeben. Ob die Schnecke im einzelnen Moment erregt wird oder nicht, hängt vom Zufall ab. Die Wahrnehmung von Reibegeräuschen aber ist für Tiere und Menschen von gleicher Bedeutung, wie die von Tönen. Man könnte erwarten, daß für die Reibegeräusche ein besonderes Organ zur Verfügung steht: das Bogengangsystem ist hierfür hervorragend geeignet. Jede + oder - Phase einer Schallwelle - sofern sie Druckdifferenzen im häutigen Vorhofslabyrinth hervorbringt - reizt die Kristen, gleichgültig, ob es sich um kurze oder lange Wellen handelt, ob annähernd gleich lange oder stark differente Wellen einander folgen, ob infolge von Interferenz komplizierte Schwin- 
gungsgebilde vorhanden sind oder einfache, normal ablaufende Phasen. Cupula und Hörhaare reagieren auf jeden noch so bunten Szenenwechsel. - Hiermit in Einklang steht die Beobachtung, daß regelmäßige, einfache Tonschwingungen verschiedener Höhenlagen gleichartige Geräuschempfindungen hervorbringen können. - Auch eine anatomische Tatsache spricht für eine akustische Funktion der Bogengänge: Das Vorhandensein der Perilymphkanäle. Für Remanenzzwecke sind sie überflüssig, nicht aber für akustische.

Die Reizung der Kristen durch die regelmäßigen Schwingungen eines Tones ist schwach. Man muß daher nach einem Momente suchen, welches die starke Reizung der Kristen durch Reibegeräusche erklärt. Die Annahme liegt nahe, sie auf Haarverschiebungen zurückzuführen, die mit stark und ruckweise wechselnder Geschwindigkeit vor sich gehen. - Bei einfachen Tönen geschieht die molekulare Versehiebung während einer Halbschwingung zwar auch mit einer von $\mathrm{O} \mathbf{z u}$ einem Maximum ansteigenden Geschwindigkeit. Aber die Übergänge erfolgen - wenn man sich die einzelnen Phasen in viele aufeinander folgende Momente zerlegt denkt allmählich. In der Bewegungsform nichts Ruckweises, Springendes. Anders bei Reibegeräuschen. Auf eine vielleicht halb abgelaufene + I Phase pfropft sich unvermittelt eine andere $+\mathrm{I}$ auf; die ist noch nicht beendet: da setzt jäh eine starke - I Phase, während die zu den ersten + I gehörigen - Phasen, die der - I korrespondierende + Phase durch Interferenz vernichtet wurden. Jähes, ruckweises, völlig regelloses Hin- und Herzittern der Hörhaare ist die Folge. Solche Bewegungen der Hörborsten wirken vielleicht energischer auf die Hörzellen, reizen ihr Plasma in höherem Grade, als das regelmäBig ablaufende Verschiebungen - vom Charakter einfacher Tonschwingungen - vermögen.

Die Differenzierung der f-, h-, sch-Geräusche in der Empfindung ist nicht sehr ausgesprochen. Analysiert man verschiedenartige, in der Natur vorkommende Reibegeräusche hinsichtlich der in ihnen enthaltenen Empfindungselemente, so sind fast immer alle drei Kategorien nachweisbar. Der Charakter der Gesamtempfindung wird bestimmt durch das Überwiegen einer, das gleichstarke Vortreten zweier oder aller drei Komponenten. Hinsichtlich der Art, wie eine Differenzierung der drei Empfindungen erfolgen könnte, sind einige Anhaltspunkte vorhanden in der Anatomie der Kristen, dem 
stärkeren Vortreten sch-artiger Geräusche bei Einwirkung einfacher Töne der 3. und 4. Oktave, sowie in der etwas verschiedenen, auf die einzelnen Kristen wirkenden Druckdifferenz.

Die an verschiedenen Stellen gleichzeitig auftretenden, einem Reibegeräusch zugrunde liegenden Wellenfragmente können vorwiegend kurzen oder langen oder mittleren Wellen angehören. Der Charaktex des durch Interferenz entstehenden Schwingungsgebildes wird danach etwas verschieden ausfallen. Bei einer Anzahl zur Interferenz gelangender kurzer Wellen wird die auf das Ohr wirkende Resultante aus kleinen und sehr häufigen Verschiebungen - positiven und negativen - bestehen. Bei langen Wellen wird das "Wellenrelief" höhere und breitere Zacken aufweisen; die Verschiebungen sind vielfach stärker und wechseln weniger oft ihre Richtung.

Wirkt in Luftleitung eine + I Phase von längerer Dauer ein, bzw. ein Fragment einer solchen, so wird der Utriculus am Vorhofsgrunde alsbald etwas stärker gespannt; die Hauptverschiebung findet weiterhin in dem oberen und unteren Perilymphsystem statt. Die Endolymphverschiebung nimmt rasch an Intensität ab. Am längsten wird dann Verschiebung der Cupula vorhanden sein in der oberen Ampulle. Dort ist die Druckdifferenz am größten. Die Cupula ist nur halb so dick wie an der äußeren Ampulle und zur Verschiebungsrichtung senkrecht, d.h. am günstigsten gestellt. Die Cupula der oberen Ampulle durfte durch alle nur möglichen Kombinationen von Phasen und Phasenfragmenten etwas stärker verschoben werden.

Eine geringere Druckdifferenz (Luftleitung) trifft die Cupula der äußeren Ampulle, die zudem doppelt so dick ist wie die der oberen Ampulle. Sie wird sich stärker verschieben nur damn, wenn die Druckdifferenzen ein wenig längere Zeit die Endolymphe etwas stärker verschieben. Kompositionen aus vorwiegend kurzen Wellenfragmenten dürften hier weniger wirksam sein, ebenso solche von langen Wellen, die eine zwar anfänglich stärkere, nachher aber desto geringere Verschiebung herbeiführen.

Die geringste Druckdifferenz (Luftleitung) trifft die untere Ampulle. Da wird die Cupula nur dann stärker verschoben, wenn die Endolymphverschiebung sehr leicht vor sich geht, also bei Einwirkung von Kompositionen aus kurzen Wellenfragmenten. Wirken vorwiegend längere oder mittlere Wellen 
ein, so beschränkt sich die sehr leichte Verschieblichkeit der Endolymphe auf den Beginn der Phase, während sie späterhin schwerer vor sich geht.' Druckdifferenzen verschieben zudem die Cupula etwas weniger leicht, wie an der oberen Ampulle. Die Crista der unteren Ampulle steht leicht schräg zur Verschiebungsrichtung, bildet mit der Ampullenquerachse einen Winkel von etwa $8^{0}$ (nach einer Retzius'schen Zeichnung abgeschätzt). Eine in der Ampullenlängsachse wirkende Kraft erzeugt somit eine kleine Kraftkomponente, die die Haube in ihrer Längsrichtung zu verschieben sucht, sie auf der einen Seite dehnt, auf der anderen komprimiert.

Nimmt man für die Hörhaare der oberen Ampulle an, daß sie erst bei einer im Verhältnis stärkeren Biegung eine stärkere Erregung der Hörzellen herbeiführen, so würden hier kurze und mittellange Wellen und Wellenfragmente weniger reizen, längere Wellen dagegen stärker.

Danach wäre eine Differenzierung der verschiedenen Geräuscharten gegeben nach ihrer vorwiegenden Zusammensetzung aus kurzen, mittleren oder längeren Wellenfragmenten. Nach den Geräuschempfindungen zu urteilen, die Töne von bestimmter Höhe begleiten, würde die untere Ampulle für Geräusche von sch-, die äuBerefür solche von f-, die obere von h-Charakter bestimmt sein, bzw. von diesen stärker erregt werden, während andere Geräusche eine Crista weniger reizen w ürden. - Differenzen der Kristen in Dicke; Stellung zur Ampullenlängsachse sind für die statische Funktion gleichgültig, nicht aber für eine a kustische.

Dafür, daß ruckweise, stark in der Geschwindigkeit wechselnde Verschiebung die Hörborsten stärker erregt, könnte folgende Beobachtung sprechen:

Lasse ich $\mathrm{c}^{2}$ vor einem $\mathrm{Ohr}$ ertönen, so höre ich neben dem Ton ein $f$-Geräusch, ebenso bei $a^{1}$. Beim Zusammenklang beider wird die Geräuschempfindung stärker. Allerdings könnte das auch bedingt sein durch Summation der von $\mathrm{c}^{2}$ und $\mathbf{a}^{1}$ ausgelösten Geräuschempfindungen. - Ähnliches tritt ein beim Zusammenklang anderer Gabeln, z. B, von $a^{1}$ mit $\mathrm{c}^{3}$ oder $\mathrm{c}^{1}$. - Bei einem dissonanten Tonintervall, z. B. a $^{1} h^{1}$, ist die Geräuschempfindung noch stärker. Die bei Dissonanzen stärker auftretende Geräuschempfindung ist eins der diese charakterisierenden Momente. Bei dissonanten Tönen aber sind diejenigen Momente

Archiv f. Ohrenheilkunde. Bd. 93. 
wesentlich stärker vorhanden, die die Kristen in erheblicherem Maße reizen könnten, als bei Konsonanzen: das sind die Zeiten, in denen die + I Phasen beider Töne einander unmittelbar folgen ${ }^{1}$, ein Vorgang, mit dem schnelle Wechsel in der Verschiebung des Labyrinthinhaltes verbunden sind, besonders wenn, wie bei den Dissonanzen, mehrere solcher kombinierter + I Phasen in wechselndem gegenseitigen Verhältnis einander folgen.

Die Klangempfindung eines einzelnen Tones wird beeinflußt durch Stärke und Art der in ihm enthaltenen Geräuschempfindungen. In manchen Klängen sind sie stark enthalten, in anderen weniger. Zahl, Art der Obertöne können das mitbedingen neben den durch das Streichen des Instrumentes, durch die Reibung des Luftstromes direkt erzeugten Reibegeräuschen. Manche, auf dem Klavier angeschlagene Tonkombinationen sind auffallend stärker von Geräuschempfindungen begleitet als andere.

Der Bogengangsapparat bietet ein gewisses Analogon zu den Weiß empfindenden Fasern der Netzhaut. Diese werden vorzugsweise erregt durch zusammengesetztes, aus Schwingungen verschiedener Länge bestehendes Licht, weniger durch einfarbiges Licht. Die Kristen werden gereizt in $m$ äßigem Grade durch einfache, regelmäßige Tonschwingungen, stark durch regellose, komplizierte Schwingungsgebilde. Dem Farbensehen entspricht gewissermaßen das Tongehör. (Die Existenz Weiß empfindender Fasern ergibt sich aus der guten Empfindlichkeit der periphersten Netzhautteile für Weiß, während sie dort für Gelb, Blau schwächer ist, für Rot, Grün ganz fehlt.)

Die Cupulaverschiebung ist am stärksten in der Mitte und auf der Höhe der Kristenrundung, am schwächsten in den abhängigsten Partien des Randes. Dadurch ist Differenzierung der Intensität von Geräuschempfindungen gewährleistet. Ganz schwache Kräfte bewegen nur die Haare der erstgenannten, sehr starke auch die der letzterwähnten Zone.

In Knochenleitung (geschlossene Gehörgänge) werden Reibegeräusche stärker gehört als bei offenen, wovon man sich durch Intensitätsvergleichung der mit dem eigenen Munde hervorgebrachten Geräusche überzeugen kann. Der durch eine + I im Vorhofe entstehende Überdruck erniedrigt sich

1) Vgl. Über die Tätigkeit des menschl. Hörorgans. A. f. O. Bd. 90. 
bei Knochenleitung, geschlossenen Gehörgängen im wesentlichen am Vorhofsgrunde, also genau so wie in Luftleitung, bei Knochenleitung, offenen Gehörgängen dagegen auch am ovalen Fenster durch Auswärtsbewegung des Steigbügels. Die Zufuhr des Druckes erfolgt durch die sich um eine geringe Größe einwärts verschiebenden Knochenwände des Labyrinths. Der in den Bogengängen entstehende Druck $(+I)$ kann sich nur durch Flüssigkeitsverschiebung nach dem Vorh ofe hin erniedrigen. Der jeweils stärkere Überdruck herrscht in den Bogengängen, der geringere im Vorhofe.

Die Bogengangsflüssigkeit verschiebt sich $(+\mathrm{I})$ an beiden Enden jeden Ganges nach dem Vorhofe; an den glatten Enden tut dies im wesentlichen nur die Endolymphe, an den Ampullen auch die Perilymphe.

Während in Luftleitung die Wasserverschiebung in beiden oberen Ampullen während der + I bogengangwärts gerichtet ist, ist in Knochenleitung das Umgekehrte der Fall. Da Biegung der Hörborsten nach der einen oder anderen Seite als akustisch gleichwertig anzusehen sein dürfte, so ist dieser Unterschied belanglos.

Der. während einer $+\mathrm{I}$ in den Bogengängen entstehende Überdruck erniedrigt sich bei offenen Gehörgängen leicht durch Perilymphverschiebung an allen drei Ampullenöffnungen. Die Endolymphverschiebung an diesen Öffnungen, damit die Cupulabiegung, Hörwirkung, fällt dann geringer aus. Bei geschlossenen Gehörgängen geschieht die Perilymphverschiebung an der unteren Ampullenöffnung ebenfalls leicht, wesentlich schwieriger aber an den Öffnungen der beiden oberen Ampullen. Entsprechend dem höheren Druck in den Bogengängen wird an diesen Stellen die Endol ym ph verschiebung stärker. Der höhere Druck im Perilymphraum oberen Bogenganges wird auch nach dem des unteren Bogenganges hin fortgeleitet, in dessen Perilymphraum, namentlich in dem der Ampulle benachbarten Teil, Überdruck entsteht im Verhältnis zu dem Druck im häutigen Kanal. Dieser wird ein wenig verengert, die Endolymphbewegung in ihm verstärkt.

Luftschallwellen wirken 1. durch den Leitungsapparat und 2. durch Luft-Knochenleitung. Während einer + I verschiebt sich die Endolymphe der Bogengänge infolge von

1. bogengangwärts an den oberen Ampullen, vorhofswärts an der unteren Ampulle; 
infolge von

2. vorhofswärts an allen drei Ampullen.

Das Endresultat der Endolymphverschiebung ist an den oberen Ampullen gleich der Differenz, an der unteren gleich der Summe der durch 1. und 2. veranlaßten Verschiebungen. Der Einfluß dieses Momentes auf die Qualität der Geräuschempfindung dürfte nicht sonderlich groß sein, da Reibegeräusche durch Luft-Knochenleitung allein viel schlechter gehört werden als durch 1 . und 2. zusammen.

Die Differenzierung der Geräusche bei Knochenleitung (geschlossenen Gehörgängen) erfolgt in ähnlicher Weise wie in Luftleitung. Zunächst verschieben sich in den + I Phasen die Endolymphteilchen der beiden oberen häutigen Ampullen und der glatten Bogengangsenden stärker, nachher erst die der häutigen unteren Ampulle. Ehe relativ stärkere Verschiebungen der unteren Cupula möglich sind, ist der Utriculus schon etwas stärker gespannt, wenn lange oder mittellange + I Phasen eingewirkt haben. Auch hier dürfte die untere Ampulle stärker nur auf kurze + I Phasen bzw. auf Fragmente solcher reagieren. Auf die äuBere Cupula. wirken, ihrer Dicke wegen, kurze + I Phasen weniger, ebenso lange, wegen relativ zu starker Spannung des Utriculus.

Für statische Zwecke sind die Perilymphkanäle überflüssig, nicht aber für akustische. Fehlten die Perilymphkanäle, wären die häutigen Kanäle unmittelbar vom Knochen umgeben, so würde bei Knochenleitung der gesammte, in den Bogengängen entstehende Druck der + I Hauben und Haare verschieden. Schädigung dieser wäre schwerer zu vermeiden. - Die Differenzierung von Reibegeräuschen wäre eine beschränkte, da stärkere Anspannung des unteren Utriculússegmentes bald nach Beginn kräftiger und etwas länger dauernder $+\mathrm{I}$ Phasen eintreten würde. Die Konstruktion von Utriculus und Bogengängen ist hervorragend gut für akustische Zwecke geeignet.

Ich schließe hier an die akustische Reizung der Macula utriculi bei Knochenleitung, geschlossenen Gehörgängen. Der Überdruck $(+I)$ in den Bogengängen und im oberen Vorhofsraum bedingt Perilymphverschiebung nach dem Vorhofsgrunde, Endolymphbewegung nach dem unteren Utriculusabschnitt. In den Bogengängen ist der Druck stets größer als im Vorhof. Aus den häutigen Bogengängen kommt sonach die Hauptverschiebung, aus dem Recessus entleert sich der etwas geringere Druek schwieriger. 
Nur bei Einwirkung sehr kurzer Tonwellen ist der untere Utriculusabschnitt so wenig gespannt, daß er während der ganzen Dauer einer $+I$ sich leicht verschieben kann. Ist er ein wenig stärker gespannt, dann reicht die Druckentleerung aus dem Recessus nicht mehr aus zur genügenden Umgestaltung der Deckhaut.

Wenn bei absoluter Taubheit die Kristen noch auf Remanenzreize reagieren, so kann das z. B. folgendermaßen bedingt sein: Beim Bestehen einer Cupula kann leichte Verdichtung derselben ihre Verschieblichkeit ein wenig herabsetzen. Dadurch könnte Bewegung der Cupula unmöglich werden bei Einwirkung der schwachen, kurzen Schallreize, aber noch stattfinden auf die etwas stärkeren, länger dauernden statischen Reize hin. Werden durch den SchrumpfungsprozeB die Hörhaare gebogen, so könnten sich subjektive Geräusche hinzugesellen. Fehlte eine Cupula, so könnte leichte Verdickung der freien Hörzellenfläche die Wirkung der Hörborsten auf den Zelleib so beschränken, daß sie nur noch bei statisehen Reizen aufträte.

Die Nervenèndstellen des Vorhofs sind gegen Schädigung durch zu starken Schall viel besser geschützt wie die Schnecke. Man versteht, weshalb starke Sehalleinwirkungen die Nervenendstellen des Vorhofs anscheinend nur wenig, das Cortische Organ und die Schneckennerven aber stark schädigen. Im Vorhof wirkt ein erheblich geringerer Teil der Kraft auf die Organe ein als in der Schnecke. Anspannung einzelner Teile des Sacculus, Utriculus verhindert eine übermäßige Inanspruchnahme der Apparate. Daraus, da $B$ die Vorhofsnervenstellen nach Einwirkung starken Schalles anscheinend nur geringe pathologische Veränderungen aufweisen, ist kein Schluß erlaubt auf ihre Nichtbeteiligung am Hörakt.

Da sowohl Drehbewegungen des Kopfes oder Körpers, wie Schallwellen Verschiebungen an dem Inhalt des Bogengangsapparates hervorbringen, so ist Erregung desselben durch statische und akustische Reize verständlich: - Die Darlegungen über die akustische Erregung des Bogengangsapparates haben nur so weit Geltung, als alle Verhältnisse den menschlichen ähnlich sind. Das ist der Fall bei vielen Säugern und Vögeln. (Weiter herunter im Tierreich habe ich die Verhältnisse nicht geprüft.)

Kalischer hat (Arch. f. Anat. u. Physiol. 1909) Dressurversuche an Hunden beschrieben. Sie durften nur fressen, 
wenn ein bestimmter Ton, der ,FreBton", auf einem Harmonium erklang, mußten mit dem Fressen aufhören, wenn ein anderer Ton angeschlagen wurde. Dieses - das leichtere — wird "Tonunterscheidungsdressur" genannt. Die Hunde durften erst wieder zugreifen, sowie der Freßton von neuem angegeben wurde. Dies - schwerere - Dressurstück ist die „Tondressur". Die Tondressur blieb für hohe und tiefe Töne gleichmäßig erhalten, wenn von einer Schnecke - das zweite Labyrinth wurde stets ganz vernichtet - nur Teile, gleichgültig welche, - durch Anbohren - zerstört wurden, andere, entweder die an der Basis oder die an der Spitze liegenden, erhalten geblieben waren.

Um, ,Ton"empfindung kann es sich dabei nicht oder nur in beschränktem Maße gehandelt haben. Waren die Spitzenteile vernichtet, die nervösen Elemente der Basiswindung gut erhalten, so konnten hohe Töne gehört werden, tiefe dagegen nicht. In einem Fall waren die nervösen Elemente in der Spitzennähe unverletzt; die Basiswindung war durchweg mit Bindegewebe ausgefüllt. Für den normalen Spitzenteil fehlte eine Stelle, an der Über- oder Unterdruck sich umsetzen konnte in Verschiebung. - Die Hunde, die nach der Operation noch Tondressur zeigten, reagierten denn auch zum Teil nicht mehr auf Pfiff und Zuruf; man konnte sie für taub halten, - so den zuletzt erwähnten Fall.

Ich habe auf einem Harmonium die die einzelnen Töne begleitenden Geräuschempfindungen geprüft und dabei folgendes gefunden: Von $\mathrm{f}^{3}$ bis $\mathrm{c}^{3}$ überwiegt die sch-Empfindung; daneben treten $\mathbf{h}$ - und namentlich $\mathbf{f}$-Empfindungen auf. Die sch-Empfindung nimmt - im allgemeinen - nach der Tiefe hin stetig $a b$. In $g^{2}$ tritt $f$ etwas stärker hervor; in $c^{2}$ sind die $\mathrm{f}-$ und sch-Empfindung etwa gleich. In $\mathrm{g}^{1}$ tritt die sch-Empfindung mehr zurück. In $\mathbf{c}^{1}$ machen sich $\mathrm{h}$ - und f-Geräusche geltend. Von hier an werden die Töne, in nach unten zunehmendem Maße, unruhig. In den tiefsten Lagen (piano) klingt der Ton direkt unterbrochen. Die Geräuschempfindung dürfte dementsprechend sein. In $c$ tritt $h$ stärker vor neben $f$. Bei den tiefsten Tönen sind stärkere, deutlich unterbrochene f- und sch-Geräusche vorhanden, die vielleicht auf erheblichere molare Luftbewegungen zurückzuführen sind. Die einzelnen Skalenbezirke unterscheiden sich also deutlich der entstehenden Geräuschempfindung nach voneinander. Diese wechselt sowohl der Qualität nach, wie hinsichtlich der Kontinuität. Ein schnecken- 
tauber Mensch würde es lernen, die einzelnen Tonbezirke des Harmoniums nach den von ihm ausgelösten Geräuschempfîndungen leidlich richtig einzuschätzen. Vielleicht könnte er auch hier und da einzelne Töne der Geräuschempfindung nach von Nachbartönen unterscheiden.

Viele Tiere unterscheiden Geräuschempfindungen vielleicht feiner als der kultivierte Mensch. Der Hund könnte die mit Tönen verbundenen Geräuschempfindungen schärfer, mehr vom Ton getrennt wahrnehmen als wir. Differenzierung der mit jedem Ton verbundenen Geräuschempfindung läge nicht ganz außerhalb des Bereichs der Möglichkeit. - Manche Töne unterscheiden sich zudem von anderen durch zufällige kleine Nebengeräusche. - Der Hund kann ferner sehen, wo eine Taste niedergedrückt wird. Fr kann sich den Ort am Harmonium merken, von dem aus der Freßton erzeugt wird. Beim Gelingen der Dressur spielen endlich eine Rolle unwillkürliche Bewegungen, ein bestimmter Gesichtsausdruck des Untersuchers beim Anschlagen des Freßtones. Viele Tiere sind äuBerst genaue Beobachter und dem Menschen nicht so selten in diesem Punkte weit überlegen. Ich erinnere hier nur an die genaue Beobachtung, die das Pferd z. B. seinem Reiter, einer ihm unbekannten Gegend zuteil werden läßt.

Bei totaler Zerstörung der Schnecke war die Tondressur. aufgehoben, nur noch die Tonunterscheidungsdressur mehr oder weniger erhalten. Bei einer derartigen Operation, leidet auch der Vorhof. Die feine Differenzierung der Geräuschempfindungen wird geschädigt. Geht der Freßton in einen anderen über, so wird die vorhandene Geräuschempfindung momentan unterbrochen; das fällt dem Tier auf. Oder beide Töne klingen einen Moment zusammen. Dann wird die Geräuschempfindung verstärkt und qualitativ verändert. Diese Änderung ist auch beim Anschlag von Nachbartönen erheblich und ins Gehör fallend. Gleichzeitig wird dann die Empfindung unruhig. Das erklärt die "Tonunterseheidung" seitens einzelner Tiere, deren Vorhof weniger, gelitten hatte.

Bei intaktem Vorhofe ist der Vorgang vielleicht folgender: Bei einem bestimmten Tone hört der Hund ein bestimmtes Geräusch, dessen Charakter entweder dem Geräusch des Freßtons ähnlich ist oder von ihm abweicht. Im ersten Fall überzeugt er sich durch raschen Blick auf die die Taste niederdrückende Hand, auf das Gesicht des Untersuchers, ob es wirklich der Freßton ist. Am geschädigten Vorhofe ist die Geräuschdifferenzierung nicht mehr von genügender Fein- 
heit. Das dem engeren Bezirk charakteristische Geräusch, in dem der FreBton liegt, wird nicht mehr so exakt wie früher aufgefaßt. Damit entfällt der Anreiz zur näheren Orientierung durch den Gesichtssinn ${ }^{1}$ ).

Vermittelt der Vorhof die Empfindung der höchsten Töne und der Reibegeräusche, dann ist er auch an der Auffassung der Sprache erheblich beteiligt. Die Wahrnebmung der Laute $f, w, h$, ch, sch, $j, s$ erfolgt vorwiegend durch den Vorhof. Wenngleich die meisten Sprachlaute Reibungsgeräusche aufweisen, die alle drei Empfindungskomponenten enthalten, solche von sch-, f-, h-Charakter, so zeigt Beobachtung der eigenen, mit Flüsterstimme hervorgebrachten Laute doch erhebliche Unterschiede im Gesamteindruck hinsichtlich der Geräuschempfindung. Die Hauptvokale weisen durchweg etwas verschiedene Reibegeräusche auf.

In $\mathrm{i}$ ist vorhanden: f stärker, daneben $\mathrm{h}$; sch wenig.

" e , ",

$\because$ a $" \quad$,

" $\quad, \quad "$

" $\mathrm{u},, \quad$, $h$ stärker, daneben $f$; sch wenig.

$\mathbf{h}, \mathbf{f}, \mathrm{sch} ; \mathbf{h}, \mathbf{f}$ annähernd gleich stark: sch etwas ïberwiegend. Die Geräuschempfindung ist leicht vibrierend.

$h, f$, sch schwächer als in a; sch weniger vortretend.

$h$; sch schwächer; $f$ gering.

Ein schneckentauber Mensch könnte es vielleicht lernen, die Vokale (in der Aussprache eines bestimmten Menschen) mit seinem Vorhof leidlich richtig aufzufassen. - Vor Jahren hörte ich auf einem Spaziergange zeitweise ein lautes, deutliches , $A^{\text {c, }}$ jedesmal eine etwas längere Zeit anhaltend. Es hörte sich an, als ob eine Anzahl Menschen laut „A" riefen. Ich ging dem Schall nach, fand als Ursache: zeitweises Ausströmen von Dampf aus einem Rohr, das unter starken Reibegeräuschen erfolgte. In der Nähe wurde der A-Charakter undeutlich wegen zu großer Stärke der Geräusche. Für den A-Laut aber sind die Reibegeräusche - neben dem Resonanz-

1) Nach Entfernung der Schläfenlappen des Gehiras (ganz: Kalischer; zum gröbten Teil: Rothmann; Sitzungsber, d. Berl. otol. Gesellsch., A. f. O. Bd. 91, S. 83,84 ) bleibt bei Hunden das absolute Tongehör (Tondressur) exhalten. Hierbei handelt es sich vielleicht ebenfalls nicht um Tonempfindung, sondem um Geräuschwahrnehmung. Daß Reibegeräusche getrennt von den Tönen im Firn wahrgenommen werden, ist nicht unwahrscheinlich. Ihr Wahrnehmungsort dürte beim Hunde, wenn die Ergebnisse von $K$ allis cher sich bestätigen, nichtim Schläfenlappen selbst liegen, sondern in dessen Nähe. 
ton - besonders charakteristisch. Die Erkennung von $\mathrm{r}$ aus den Reibegeräuschen ist leicht; es werden stark unterbrochene Geräusche von f- und h-Charakter gehört. Das (deutsche) 1 enthält vorwiegend ein - etwas unruhiges - h-Geräusch. Es könnte mit e verwechselt werden. $m, n$ sind von ähnlichen Geräuschen wie a begleitet. b, p, d, t, g, k dürften der Geräuschempfindung nach schwer zu unterscheiden sein. - Namentlich für die Auffassung der Flüstersprache dürfte der Vorhof ein unterstützendes Moment abgeben.

Die wiederholt beobachtete Hör ver besserung für Sprache nach Steigb ügelentfernung bei unbeweglichem Verschluß beider Fenster beruht auf folgendem: Hörerregend wirken nur die Schallwellen, die aus Luft auf den Schädel übergehen. Das ovale Fenster wirkt als Druckausgleichstelle. Die akustische Funktion der Bogengänge dürfte unter diesen Bedingungen ziemlich ungestört vor sich gehen, die des Sacculus und Utriculus beeinträchtigt sein. Befindet sich im ovalen Fenster eine dünne, leicht bewegliche Membran, so ist die Verschiebungsmöglichkeit da größer, als wenn der normale Steigbügel vorhanden ist. Damit ist auch die Möglichkeit etwas stärkerer Erregung der Schnecke gegeben. Für die Bewegung der Schneckenzwischenwand ist maßgebend der während der + I Phase in der Paukentreppe herrschende Überdruck $^{\mathbf{1}}$ ). Bedingung für etwas stärkere Erregung der Schnecke ist, daß die durch molekulare Verschiebung der übrigen Labyrinthwände (+ I) erzielte Auswärtsbewegung der Verschlußmembran ovalen Fensters diese nur mäßig spannt, daß also der Überdruck in der Paukentreppe keinen großen Widerstand findet.

Die Qualität der Tonempfindung wird allerdings erheblich differieren gegenüber der bei nor maler Schneckenreizung. Während einer + I Phase würden hohe Töne an der Grundhaut nach der Spitze hin abnehmende Verschiebungen zuwege bringen, wegen der steigenden Wirkung des Helikotrema. An der Basis träte daher Tonempfindung ein, die spitzenwärts allmählich schwächer wird, schließlich ganz aufhört. Je tiefer ein Ton, um so größere Teile der Schnecke würde er von der Basis nach der Spitze hin zur Tonempfindung erregen. - Differenzierung verschiedener Tonhöhen ist dabei wohl möglich. Nicht anderes geschieht, wie beim normalen Hören eines Tones: Zusammenfassung differenter

I) A. f. O. Bd. 89 , S. 249. 
Einzelempfindungen zu einer Gesamtempfindung, nur in wesentlich vergrößertem Maßstabe, namentlich für mittlere und tiefere Töne. Notwendig freilich ist vermehrte Aufmerksamkeit, um die verändert klingende Sprache zu verstehen. Hinsichtlich Auffassung der Sprache aber hat jeder Mensch. eine erhebliche Übung. da nicht nur die verschiedenen Dialekte sehr voneinander abweichen, sondern auch der Klang der Sprache bei den einzelnen Menschen.

Ich fasse das Ergebnis zusammen in folgende Sätze:

Neben statischen besitzt der menschliche Vorhof akustische Funktionen.

Die Maculae bieten gute Bedingungen für die Wahrnehmung höchster Töne, die Cristae für die Empfindung von Reibegeräuschen mit sch-, f-, h-ähnlichem Charakter.

Akustische Erregung der Maculae kommt, unter bestimmten Bedingungen, zustande durch Umgestaltung der Deckhäute. Diese wird erzeugt durch Druckdifferenzen, die im Vorhofe während der + I Phasen entstehen. Das deckhautverändernde Moment ist: stärkerer Druck über den Maoulae, geringerer seitlich daneben, wodurch die Deckhaut sich nach der Unterdruckzone hin abflacht und verbreitert.

Akustische Erregung der Cristae beruht auf rascher Verschiebung und Biegung der Cupulae mit den Hörhaaren durch Druckdifferenzen, die sich vom Vorhofe aus in die Bogengänge hinein geltend machen. 